\section{AB0687 ASSOCIATION BETWEEN GOLIMUMAB TAPERING STRATEGY AND DRUG SERUM LEVELS IN SPA-PAZ SPONDILOARTHRITIS COHORT}

C. Redondo ${ }^{1}$, A. Martínez ${ }^{2}$, C. Plasencia ${ }^{1}$, V. Navarro-Compán ${ }^{1}$, L. Nuño ${ }^{1}$, D. Peiteado ${ }^{1}$, A. Villalba ${ }^{1}$, A. Jochems ${ }^{2}$, D. Pascual-Salcedo ${ }^{2}$, A. Balsa ${ }^{1}$.

${ }^{1}$ Rheumatology; ${ }^{2}$ Immunology Unit, la Paz University Hospital, Madrid, Spain

Background: Golimumab (GIm) is a tumour necrosis factor (TNF) inhibitor used as Spondiloarthritis $(\mathrm{SpA})$ treatment. There is more and more evidence of tapering therapies used in maintained low disease activity patients, avoiding overtreatments and adverse effects. Up to now, there are no studies concerning SpA patients with Glm tapering therapy and serum drug levels measured prior to tapering strategy.

Objectives: To analyze clinical and analytical course of SpA patients with Glm interval prolongation in SpA-Paz cohort. Associate pre-tapering Glm serum levels with flare occurrence along tapering treatment.

Methods: Observational prospective SpA-Paz cohort study of 22 patients treated with Glm that remained with low disease activity, so they started tapering strategy (Tap-S). We measured clinical activity with ASDAS and $\triangle$ ASDAS at baseline, pretapering visit (v-pre)and at 6,12, 18 and 24 months after tapering starting (v-end). All of them had a clinically important improvement determined as $\triangle$ ASDAS $>1$ '1. We registered flares, defined as clinical worsening based on patient anamnesis and physical examination and on diverse activity indexes made from the beginning until the last visit. Drug serum levels in v-pre were measured by ELISA and classified following optimal concentration previously defined in our laboratory: $<0^{\prime} 7 \mathrm{mg} / \mathrm{L}, 0^{\prime} 7-1^{\prime} 4 \mathrm{mg} / \mathrm{L},>1^{\prime} 4 \mathrm{mg} / \mathrm{L}$. We used the software Graph-Pad Prism 6 for statistical analysis.

Results: 22 of 79 patients from our cohort $(28 \%)$ initiated Tap-S. The age range was between 25 and 70 years, $73 \%$ men and $27 \%$ women. $52 \%$ were non-smokers. 5 patients $(23 \%)$ received another antiTNF drug previously $(4$ Infliximab, 1 Etanercept). All of them had a 6 months visit prior to tapering, 18 patients had 12 months visit, 8 had 18 months visit and 5 had 24 months visit. ASDAS and $\triangle$ ASDAS were similar in v-pre and v-end (ASDAS: 1'3 30 ' $4 \mathrm{v}$-pre vs 1'2 20 0'7 v-fin, $p=0$ '3; $\triangle$ ASDAS: 2'3 \pm 0 '7 v-pre vs 2' $4 \pm 0$ ' 9 v-end, $p=0$ ' 6 ). 13 patients $(59 \%)$ had 1 or 2 flares along tapering therapy. The occurrence of flare average was 14 months. In all the patients with flares, disease activity was controlled returning to previous tapering dose $(4 / 13,31 \%)$, to monthly schedule $(6 / 13,46 \%)$ or remaining tapering dose with symptomatic treatment adjustments $(3 / 13,23 \%)$. None of them discontinued the treatment. At v-end, ASDAS and $\triangle$ ASDAS were similar between patients with and without flares along Tap-S (ASDAS v-end: $1 \pm 0^{\prime} 7$ in patients without flares vs $1^{\prime} 3 \pm 0^{\prime} 7$ in patients with flares, $\mathrm{p}=0^{\prime} 4, \triangle$ ASDAS $v$-fin: $2^{\prime} 7 \pm 0^{\prime} 7$ in patients without flares vs $2^{\prime} 2 \pm 1$ in patients with flares, $\left.p=0^{\prime} 3\right)$. All the patients $(4 / 4,100 \%)$ that were under optimal drug concentration $\left(<0^{\prime} 7 \mathrm{mg} / \mathrm{L}\right)$ had at least one flare along Tap-S; however, only $23 \%$ (3/9) of those who were in optimal drug concentration in v-pre (0'7-1'4 mg/L) had flares.

Conclusions: The tapering strategy in our SpA cohort treated with GIm results in similar disease activity control after 24 months of monitoring. The activity disease in patients with flares was controlled with symptomatic or biologic treatment adjustments avoiding therapy discontinuation. Flares incidence was more frequent in patients under optimal drug concentration in v-pre.

Disclosure of Interest: None declared

DOI: 10.1136/annrheumdis-2017-eular.5911

\section{AB0688 EFFECT OF TREATMENT WITH NON-STEROIDAL ANTI-INFLAMMATORY DRUGS ON DISEASE ACTIVITY IN PATIENTS WITH EARLY AXIAL SPONDYLOARTHRITIS BASE ON DATA FROM 2 YEARS FOLLOW UP OF CORSAR COHORT}

D. Rumiantceva, T. Dubinina, O. Rumyantseva, A. Demina, E. Agafonova, S. Erdes, S. Krasnenko. Laboratory of spondyloarthritis, Nasonova Research Institute of Rheumatology, Moscow, Russia, Moscow, Russian Federation

Background: Currently non-steroidal anti-inflammatory drugs (NSAIDs) are the first-line drugs for treatment of axial spondyloarthritis (axSpA) and much discussed question of influence frequency of intake NSAIDs on axSpA disease activity. Objectives: To compare the frequency of intake NSAIDs with early axSpA disease activity.

Methods: The research included 65 patients (pts) with axSpA (criteria ASAS 2009) with disease duration $<5$ years and age at onset $<45$ years. Pts at least 2 years follow up, $32(49,2 \%)$ males, pts mean age was $28,5(5,8)$ y., average disease duration - 24,1 $(15,4) \mathrm{mo}, 60(92,3 \%)$ pts were HLA-B27 positive. At baseline all pts were NSAID-naïve, DMARD-naïve, anti-TNF-naïve. For 2 years pts taking NSAIDs at therapeutic doses. The dosages of NSAIDs accounted by

Table 1. Outcome parameters at baseline and after 2 years of NSAID treatment

\begin{tabular}{lccc}
\hline & $\begin{array}{c}\text { Baseline, NSAID naïve } \\
(\mathrm{n}=65)\end{array}$ & $\begin{array}{c}2 \text { years after baseline with NSAIDs } \\
(\mathrm{n}=50)\end{array}$ & $\mathrm{p}$ \\
\hline BASDAI, mean (s.d) & $3,6(1,9)$ & $2,4(1,9)$ & 0,6 \\
BASDAI $\geq 4, \%$ pts & $29(44,6 \%)$ & $10(20,0 \%)$ & 0,05 \\
BASDAI $<3, \%$ pts & $26(40,0 \%)$ & $46(92,0 \%)$ & 0,01 \\
ASDAS-CRP, mean (s.d) & $2,4(1,1)$ & $1,7(1,1)$ & 0,0009 \\
ASDAS-CRP $\geq 2,1, \%$ pts & $39(62,9 \%)$ & $22(44,0 \%)$ & 0,4 \\
ASDAS $<1,3, \%$ pts & $11(16,9 \%)$ & $27(54,0 \%)$ & 0,004 \\
\hline
\end{tabular}

the ASAS NSAID index. 1 year after baseline $-9(13,8 \%)$ pts started treatment with anti - TNF because of high activity of the disease and absence of effect from 2 NSAIDs. 2 years after baseline number of pts with anti-TNF equal - $15(23,0 \%)$. Results: Compared with baseline characteristic after 2 years have significantly reduced disease activity and increased the number of ASAS partial remission (PR) in patients with axSpA (Table 1).

There were no statistical differences between the frequency of NSAIDs intake and early axSpA disease activity (Table 2).

Table 2. Outcome parameters at 1 and 2 years after baseline depend on NSAID index $>50$ or NSAID index $<50$

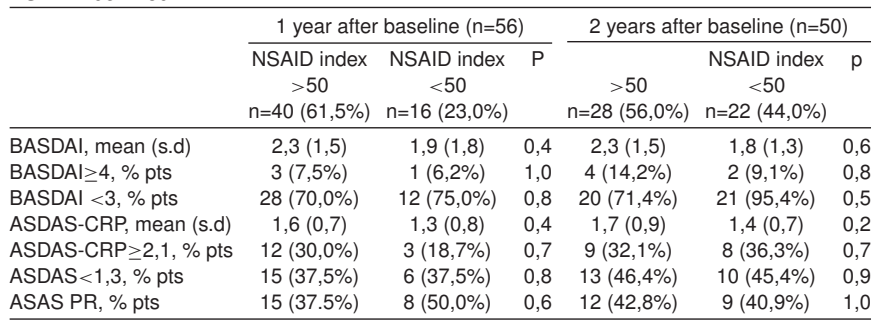

Conclusions: Long reception of NSAIDs in patients with early axSpA reduces disease activity, however, the receive frequency does not affect the activity of the disease.

Disclosure of Interest: None declared

DOI: 10.1136/annrheumdis-2017-eular.3646

\section{AB0689 METABOLIC SYNDROME IN ANKYLOSING SPONDYLITIS AND THE EFFECT OF ANTI-TNF THERAPY}

F. Atzeni ${ }^{1}$, L. Gianturco ${ }^{2}$, I.F. Masala ${ }^{3}$, S. Bongiovanni ${ }^{1}$, L. Boccassini ${ }^{1}$, P. Sarzi-Puttini ${ }^{1}$, M. Turiel ${ }^{1} .{ }^{1}$ Rheumatology Unit, L.Sacco University Hospital; ${ }^{2}$ Cardiology Unit, Department of Biomedical Sciences for Health, IRCCS Galeazzi Orthopedic Institute, Milan; ${ }^{3}$ Orthopedic and Trauma, Santissima Trinità Hospital, Cagliari, Italy

Background: Metabolic syndrome (MeS) is a cluster of cardiovascular (CV) risk factors (obesity, dyslipidemia, hypertension, alterations in glucose metabolism, and insulin resistance) whose prevalence is increasing particularly in devoloped countries. In comparison with the general population, patients with ankylosing spondylitis (AS) are at increased risk of cardiovascular (CV) disease, which is one of the main causes of mortality among them.

Objectives: The aim of this study was to identify the relationship between MeS and disease activity in AS patients, and evaluate the the effect of anti-tumour necrosis factor (TNF) therapy.

Methods: The study involved 30 outpatients who met the New York diagnostic criteria for AS: 14 males and 16 females; mean age $53.07 \pm 10.73$ years; mean disease duration $5.03 \pm 4.06$ years. All of the patients were being treated with DMARDs and anti-inflammatory drugs, but none had received any biological agents or steroids at baseline. The patients underwent laboratory tests (lipid profile and glucose levels), a clinical and standard echocardiographic examination, carotid ultrasonography (including the assessment of intima-media thickness and pulse wave velocity), and speckle tracking echocardiography (STE) of the left ventricle (LV) using Philips QLAB software to evaluate their CV risk profiles. Functional impairment and disease activity were assessed using the Bath Ankylosing Spondylitis Disease Activity Index (BASDAI) and the Bath Ankylosing Spondylitis Functional Index (BASFI). All of the patients were evaluated at baseline and after 12 months of anti-TNF treatment. The data were statistically analysis using non-parametric chi-squared and Mc-Nemar tests, and the Wilcoxon-MannWhitney test.

Results: Before starting anti-TNF therapy, the cohort was divided into two groups: those with MeS $(25 \%)$ and those without. The patients with MeS higher BASDAI scores (chi-squared test: $4.85 ; \mathrm{p}=0.03$ ). After 12 months of follow-up, there was a statistically significant improvement in global longitudinal strain (GLS) in both groups: from a baseline median of 19.94 (IQR 18.66-20.78) to a median of 21.46 (IQR 20.37-22.69) $(\mathrm{p}=0.02)$ in the non-MeS group, and from a baseline median of 18.88 (IQR 16.00-19.35) to 19.54 (IQR 18.21-19.98) ( $p=0.04$ ) in the MeS group. No other changes in CV parameters were observed. The improvement in BASDAI scores was greater in the MeS group, but the difference was not statistically significant. The prevalence of MeS decreased after 12 months of anti-TNF therapy, although the McNemar test showed that the decrease was of borderline significance $(\mathrm{p}=0.048)$.

Conclusions: AS patients are affected by MeS. The effects of anti-TNF drugs on $\mathrm{MeS}$ are still controversial, but our findings suggest that they improve CV markers such as GLS as well as disease activity. The main limitation of this study is its small sample size and short follow-up; further studies are required in order to clarify the influence of MeS on clinical therapeutic outcomes.

Disclosure of Interest: None declared

DOI: 10.1136/annrheumdis-2017-eular.1792 